\title{
The Importance of Sphingosine Kinase in Breast Cancer: A Potential for Breast Cancer Management
}

\author{
Dutt S. Patel ${ }^{1}$, Farrukh Ahmad ${ }^{2,}{ }^{3}$, Majdi Abu Sneineh ${ }^{1}$, Ravi S. Patel ${ }^{1}$, Sai Rohit Reddy ${ }^{1}$, Adiona \\ Llukmani $^{1}$, Ayat Hashim ${ }^{1}$, Domonick K. Gordon ${ }^{1,4}$ \\ 1. Internal Medicine, California Institute of Behavioral Neurosciences \& Psychology, Fairfield, USA 2. Emergency \\ Medicine, California Institute of Behavioral Neurosciences \& Psychology, Fairfield, USA 3. Emergency Department, \\ Beaumont Hospital, Dublin, IRL 4. Internal Medicine, Scarborough General Hospital, Scarborough, CAN
}

Corresponding author: Dutt S.Patel, douxli@gmail.com

\begin{abstract}
Breast cancer management includes a combination of surgery, radiation therapy, and chemotherapy. While this management has proven effective, it is not perfect. To expand the umbrella of management to resistant breast cancer tumors, researchers have explored the idea of sphingosine kinase (SphK) and sphingosine-1phosphate (S1P) as a potential target for treatment. In this article, we review the mechanism of the sphingosine kinase/sphingosine-1-phosphate (SphK/S1P) axis along with its effect on the tumor microenvironment (TME) and compounds that have been studied inhibiting the SphK/S1P axis. We searched for relevant articles in the last five years in Medline and PubMed Central. Inclusion criteria, exclusion criteria, and quality checklists were applied to identify the most relevant articles. We compiled the information that has been summarized in the respective tables and figures provided in this review. The metabolism of sphingolipids was summarized, followed by the SphK/S1P upregulation in breast cancer cells. The variety of effects by upregulation of SphK led to an increase in inflammation, growth, and metastasis in breast cancer tumors. The increase in S1P also impacted the TME, including the cells and surrounding tissue, allowing the breast tumors to thrive. The final point made was a summary of the compounds and drugs that inhibited the SphK/S1P axis. They have proven their effectiveness and show even greater efficacy in combination with docetaxel and doxorubicin in preclinical studies. In conclusion, what is known about the SphK/S1P axis within breast cancer cells is immense but incomplete as we summarize what is known so far. Having a complete picture will allow a faster transition to application in the clinical field but clinical trials have not commenced as of yet.
\end{abstract}

Received 01/10/2021 Review began 01/24/2021 Review ended 02/15/2021 Published 02/18/2021

\section{() Copyright 2021}

Patel et al. This is an open access article distributed under the terms of the Creative Commons Attribution License CC-BY 4.0., which permits unrestricted use, distribution, and reproduction in any medium, provided the original author and source are credited.
Categories: Pathology, Oncology

Keywords: sphingosine kinase, sphingosine-1-phosphate, breast neoplasms

\section{Introduction And Background}

Breast cancer is the second most diagnosed cancer in women living in the United States after skin cancer [1]. It is estimated that in $2020,30 \%$ of all cancers in women will be diagnosed as breast cancer, according to the National Breast Cancer Foundation [2]. While breast cancer mortality has decreased in the last few decades due to advances in detection and management, it is still the second leading cause of cancer death in women [3]. Decreased mortality due to better management can be linked to the identification of molecular markers, gene profiles, and targeting specific receptors. Most breast cancers are estrogen receptor (ER) and progesterone receptor (PR) positive (70\%), while some overexpress human epidermal receptor 2 (HER2). Approximately $15-20 \%$ of breast cancers are negative for all three receptors, known as triple-negative breast cancer (TNBC). This type has the worst prognosis due to aggressiveness or metastasis and lack of targeted treatment [4]. It does not respond to hormonal or antibody therapies like tamoxifen or trastuzumab, making chemotherapy the primary choice for management. Currently, such tumors are treated with a combination of surgery, radiation therapy, and chemotherapy. While this therapy can improve survival rates, many side effects reduce quality of life and even interfere with further cancer treatment [4].

To manage resistant breast cancer more efficiently, researchers have tried to tackle this issue from different angles. One angle researched over the past two decades is the role of lipid phosphates in the microenvironment of cancerous tumors [5]. Of the lipid phosphates and their corresponding lipid phosphate phosphatases (LPP), the primary focus is on how sphingosine-1-phosphate (S1P) and sphingosine kinase (SphK) upregulation can promote the growth of a tumor via angiogenesis and lymphangiogenesis [6]. By phosphorylation of sphingosine by either isoenzyme, sphingosine kinase 1 (SphK1), or sphingosine kinase 2 (SphK2), S1P is formed [5]. S1P generated by SphK1 impacts cancer progression because the S1P formed is exported out of the cell to act on its specific cell surface G-protein-coupled receptors (S1P receptors 1-5). Of the receptors, S1P receptor 1 (S1PR1), S1P receptor 3 (S1PR3), and S1P receptor 4 (S1PR4) are linked with poor prognosis [6]. The S1P acts on these receptors in an autocrine and paracrine fashion in a process called inside-out signaling [5]. The inside-out signaling causes other downstream signals to activate, which increases the growth of new blood vessels and lymphatics. This process has many names, including 
SphK/S1P, SphK/S1P/S1P receptor, or just S1P axis [4,7]. Without this axis, the tumor would not grow more than $3 \mathrm{~mm}$; all the more reason it is a primary focus for future management [6]. This specific mechanism has become a promising target for future treatment of breast cancer. Currently, novel drugs are being tested with preclinical breast cancer models that target the SphK/S1P axis [3].

While there has been growing research in this area, this mechanism's applicability for treatment is infantile. It is a relatively novel mechanism, and clarification is needed regarding its potential role in breast cancer management. We hope to shed some light as we review the SphK/S1P axis, focus on the underlying mechanism, and discuss recent developments.

\section{Review}

\section{Methods}

Search Strategy and Study Selection

PubMed was used to search for relevant publications. The search included the electronic databases Medline and PubMed Central. The keywords used in the search process included "breast cancer and sphingosine kinase," "breast neoplasm and sphingosine-1-phosphate," "breast cancer and sphingosine-1-phosphate," “(breast neoplasm and sphingosine kinase," "Breast Neoplasms”[Mesh]) AND “sphingosine kinase” [Supplementary Concept], and ("Breast Neoplasms/chemistry”[Mesh] OR "Breast

Neoplasms/enzymology”[Mesh]) AND “sphingosine kinase” [Supplementary Concept]. Articles were searched from January 2015 to November 2020 and were imported into Mendeley for organization and citations. After removing the duplicates, all titles and abstracts were reviewed using the inclusion and exclusion criteria. Subsequently, the full-text articles were reviewed, and quality assessment tools were applied for final selection. Figure 1 shows a summary of the study selection process in a Preferred Reporting Items for Systematic Reviews and Meta-Analyses flow diagram.

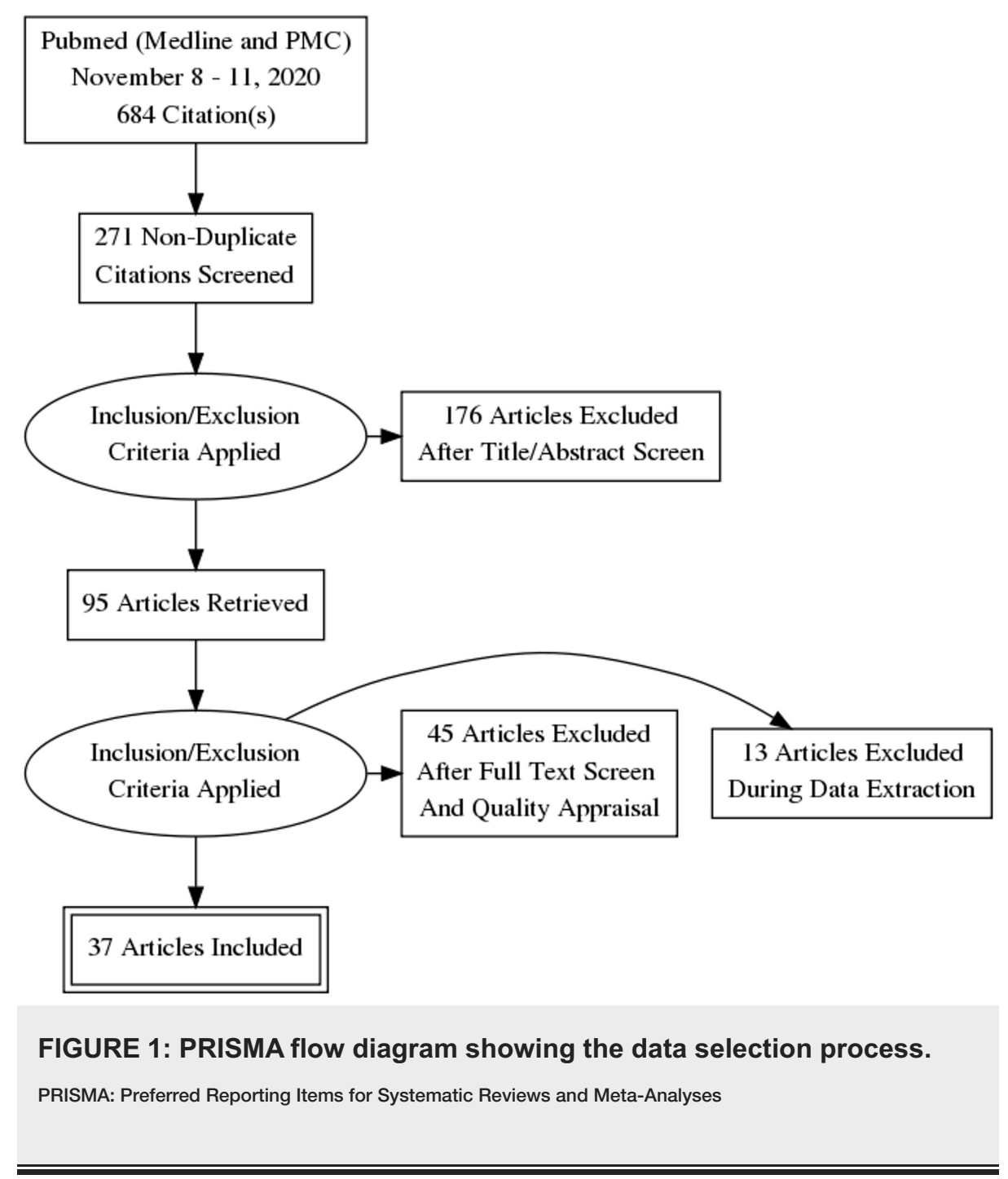




\section{Cureus}

\section{Study Selection Criteria}

The inclusion criteria consisted of: (1) All publications in the last five years (January 2015 to November 2020); (2) articles in only the English language; (3) studies originating from any country; (4) full-text articles; and (5) all studies emphasizing breast cancer and SphK/S1P.

The exclusion criteria consisted of: (1) All publications older than five years (published before January 2015); (2) articles not in the English language; (3) articles not relevant to breast cancer or SphK/S1P; (4) articles not available in full text; and (5) Gray literature.

\section{Results}

Search Results and Characteristics

Following the inclusion criteria, 37 studies were used for the review. The studies included 6,627 patient samples, multiple breast cancer cell lines, cats, and mice. Of the 37 included studies, there were 25 crosssectional studies, eight literature reviews, and four cohort studies. The quality of the studies was checked using the respective quality assessment tool and are shown in Table 1 and Table 2 . The cross-sectional studies were assessed using the Newcastle-Ottawa Quality Assessment Scale adapted for cross-sectional studies, and the minimum number of stars to qualify as "high quality" was seven. The Scale for the Quality Assessment of Narrative Review Articles or the SANRA checklist was used to assess the literature reviews. The total score available was 12 and the minimum to qualify as "high quality" was seven. The remaining cohort studies were reviewed using the Newcastle-Ottawa Quality Assessment Scale-Cohort Studies, and the minimum to qualify as "high quality" was seven.

\section{Pathophysiology of Sphingosine Kinase and Breast Cancer}

Information was taken from 27 of the 37 included articles and compiled into a flow chart (Figure 2). A summary of what is known about the normal metabolism of sphingolipids is shown in the upper left portion of the figure. Below the first part of the figure a second flow chart summarizes the pathophysiology of breast cancer cells, emphasizing the role of SphK/S1P in the growth, invasion, and metastasis of cancerous tumors. The figure will be discussed in further detail in the next section. The studies from Table 1 were used to construct Figure 2 and will be discussed accordingly.

\section{Role of Tumor Microenvironment and Promising Compounds}

Information was taken from 10 of the 37 included articles to create Figure 3 and Table 1 , outlining the significant consequences breast cancer cells have on the tumor microenvironment (TME). The findings will be discussed in the next section. Information from 16 of the 37 articles has been arranged in Table 2, summarizing the compounds or drugs that inhibit the SphK/S1P axis as well as their relevant findings. The details will be discussed in the next section.

\begin{tabular}{|c|c|c|c|c|c|}
\hline $\begin{array}{l}\text { First } \\
\text { author }\end{array}$ & $\begin{array}{l}\text { Year of } \\
\text { publication }\end{array}$ & Reference & $\begin{array}{l}\text { Quality } \\
\text { assessment } \\
\text { tool }\end{array}$ & $\begin{array}{l}\text { Quality } \\
\text { assessment } \\
\text { score }\end{array}$ & Findings/Results \\
\hline Chen, Z & 2020 & [8] & NOS-CS & 7 & $\begin{array}{l}\text { SphK1 regulated stem cell characteristics as well as migration, } \\
\text { invasion, and induced EMT in breast cancer cells }\end{array}$ \\
\hline Kim, EY & 2020 & [9] & NOS-CS & 7 & $\begin{array}{l}\text { In highly metastatic breast cancer cells, there was an increased } \\
\text { expression of both IL-22R1 and S1PR1. IL-22 enhanced the } \\
\text { expression in the receptors of breast cancer cells while also } \\
\text { increasing S1P and MCP-1 in MSCs }\end{array}$ \\
\hline Oshi, M & 2020 & [10] & NOS-C & 7 & $\begin{array}{l}\text { The Tumor Angiogenesis Score was evaluated, and a high score } \\
\text { was associated with increased expression of S1P-related genes, } \\
\text { hypoxia, increased angiogenesis, increased expression of } \\
\text { inflammation-related genes, metastatic redundancy, and } \\
\text { decreased infiltration of immune cells. A high score was not } \\
\text { associated with overall survival, aggressive features, or } \\
\text { response to neoadjuvant chemotherapy }\end{array}$ \\
\hline & & & & & $\begin{array}{l}\text { Evaluation of } 36 \text { genes related to sphingolipid metabolism was } \\
\text { completed in both breast cancer cells and healthy controls. Five } \\
\text { genes were upregulated while the S1PR1 gene was }\end{array}$ \\
\hline
\end{tabular}




\section{Cureus}

\begin{tabular}{|c|c|c|c|c|c|}
\hline $\begin{array}{l}\text { Sakharkar, } \\
\text { MK }\end{array}$ & 2020 & [11] & NOS-CS & 7 & $\begin{array}{l}\text { downregulated in breast cancer cells. The gene pair correlation } \\
\text { coefficient was high in the control group but lost their } \\
\text { correlation in breast cancer patients. No genes were identified } \\
\text { as having a strong prognostic value }\end{array}$ \\
\hline Singh, SK & 2020 & [4] & SANRA & 9 & $\begin{array}{l}\text { Summarized current therapy available forTNBC followed by S1P } \\
\text { metabolism and the potential for S1P to be a target for future } \\
\text { therapy. Importance of S1P in chemotherapy-induced peripheral } \\
\text { neuropathic pain and cancer-related bone pain was emphasized }\end{array}$ \\
\hline Zhong, L & 2020 & [12] & NOS-CS & 7 & $\begin{array}{l}\text { Lower S1PR1 expression correlated with a poor overall survival } \\
\text { in breast and lung carcinomas, while increased S1PR1 } \\
\text { expression correlated positively with immune cell infiltration }\end{array}$ \\
\hline $\begin{array}{l}\text { Acharya, } \\
\text { S }\end{array}$ & 2019 & [13] & NOS-CS & 7 & $\begin{array}{l}\text { TNBCs had a high expression of SphK1 and promoted lung } \\
\text { metastasis while blocking expression produced the opposite } \\
\text { effect. FSCN1, a contributor to metastasis, was found to be } \\
\text { upregulated by SphK1 through the activation of NFkB. By } \\
\text { inhibiting the pathway, tumor growth and lung metastasis were } \\
\text { inhibited as well }\end{array}$ \\
\hline Liu, S & 2019 & [14] & NOS-CS & 7 & $\begin{array}{l}\text { Upregulation of S1PR1 increased EDV while inhibiting VM by } \\
\text { increasing } \beta \text {-catenin expression leading to decreased VE- } \\
\text { cadherin. S1PR1 also increased VE-cadherin phosphorylation } \\
\text { promoting the separation of VE-cadherin from } \beta \text {-catenin }\end{array}$ \\
\hline $\begin{array}{l}\text { Alshaker, } \\
\text { H }\end{array}$ & 2019 & [15] & NOS-CS & 7 & $\begin{array}{l}\text { RNA transcriptome microarray technology was used to } \\
\text { understand the effects of SphK knockdown. RAS, MAPK, } \\
\text { GTPase, Wnt, and PI3K were some of the pathways which were } \\
\text { upregulated in the absence of SphK1 and SphK2, while other } \\
\text { pathways involved in cancer were unaffected }\end{array}$ \\
\hline El Buri, A & 2018 & [16] & NOS-CS & 7 & $\begin{array}{l}\text { Breast cancer stem cell lines released S1PR2 in which the } \\
\text { exosomal S1PR2 activated fibroblasts.The processing of } \\
\text { exosomal S1PR2 by fibroblasts was also identified }\end{array}$ \\
\hline Engel, N & 2018 & [17] & NOS-CS & 7 & $\begin{array}{l}\text { SGPL1 was identified in the cytoplasmic membrane. SGPL1 } \\
\text { levels were low in breast cancer cells. High SGPL1 levels } \\
\text { prevented S1P stimulation }\end{array}$ \\
\hline Shimizu, Y & 2018 & [18] & NOS-CS & 7 & $\begin{array}{l}\text { Lack of SphK1 causes a decrease in expression of claudin-2 in } \\
\text { HER2/neu-induced breast tumors as well as reduction in overall } \\
\text { tumor development. Increased SphK1 expression promoted } \\
\text { cancerous cellular functions in the same breast cancer cells }\end{array}$ \\
\hline Wang, S & 2018 & [19] & NOS-CS & 9 & $\begin{array}{l}\text { Hyperexpression of SphK1 was seen in breast cancer tissues } \\
\text { and correlated with high levels of S1P. Inhibiting SphK1 } \\
\text { decreased metastatic potential in breast cancer cell lines. } \\
\text { Increased S1P levels was identified to activate Notch signalling } \\
\text { through the S1PR3 }\end{array}$ \\
\hline $\begin{array}{l}\text { Yamada, } \\
\text { A }\end{array}$ & 2018 & [20] & NOS-CS & 7 & $\begin{array}{l}\text { Increased expression of ABCC1 transporter increased S1P } \\
\text { secretion, metastatic potential, enhanced tumor growth, and the } \\
\text { S1P exported by the transporter increased transcription of } \\
\text { SphK1. This contributed to a poor prognosis in breast cancer } \\
\text { patients. On the contrary, ABCB1 was not associated with any } \\
\text { of these effects }\end{array}$ \\
\hline Do, SI & 2017 & [21] & NOS-C & 7 & $\begin{array}{l}\text { SphK1 was proven to have overexpression in breast cancer } \\
\text { tissue compared to normal mammary tissue showing } \\
\text { association with higher metastatic potential and aggressiveness }\end{array}$ \\
\hline Calis, IU & 2017 & [22] & NOS-CS & 7 & $\begin{array}{l}\text { Lateral motility, adhesion and proliferation, and viability of breast } \\
\text { cancer cell lines were statistically reduced by silencing S1PR1 } \\
\text { and S1PR3 genes. The silencing of both genes was greater than } \\
\text { silencing either gene individually }\end{array}$ \\
\hline Maia, LP & 2017 & [23] & NOS-CS & 7 & $\begin{array}{l}\text { S1PRs in breast cancer patients and tumors were expressed in } \\
\text { lymphocytes, monocytes, and granulocytes at varying levels. } \\
\text { Variable expression of genes of sphingolipid metabolism and }\end{array}$ \\
\hline
\end{tabular}




\section{Cureus}

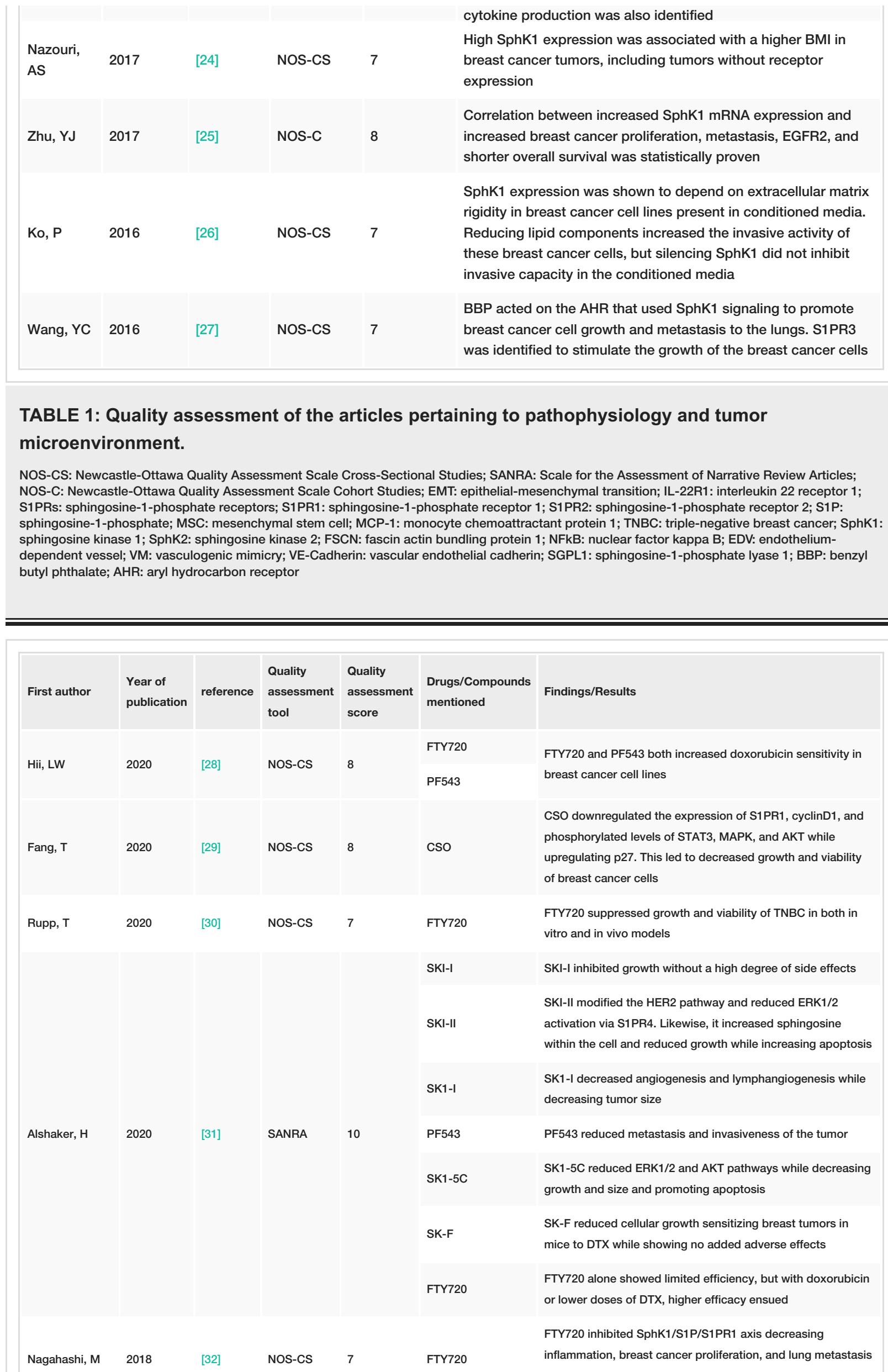




\section{Cureus}

\begin{tabular}{|c|c|c|c|c|c|c|}
\hline & & & & & & caused by obesity \\
\hline \multirow[t]{2}{*}{ Alshaker, $\mathrm{H}$} & \multirow[t]{2}{*}{2018} & \multirow[t]{2}{*}{ [33] } & \multirow[t]{2}{*}{ NOS-CS } & \multirow[t]{2}{*}{7} & $\begin{array}{l}\text { Controls: SKI-178, } \\
\text { 12aa, and SK1-I }\end{array}$ & $\begin{array}{l}\text { SKI-178, 12aa, and SK1-I are known SK1 inhibitors used to } \\
\text { find six new compounds with similar effects, SK-A, SK-B, SK- } \\
\text { C, SK-D, SK-E, SK-F (field template analysis); SK-C, SK-E, } \\
\text { SK-F inhibited SK1 in a cell model, with SK-F having the } \\
\text { highest inhibition percentage }\end{array}$ \\
\hline & & & & & $\begin{array}{l}6 \text { new: SK-A to SK- } \\
\text { F }\end{array}$ & $\begin{array}{l}\text { There was no significant change in tumor size or viability with } \\
\text { the use of SK-F alone compared to the control group, but } \\
\text { there was a significant reduction with DTX }\end{array}$ \\
\hline Geffken, K & 2018 & [3] & SANRA & 10 & FTY720 & $\begin{array}{l}\text { Phosphorylated FTY720 inhibits HDACs allowing ER to form in } \\
\text { ER-negative breast cancer. Unphosphorylated FTY720 inhibits } \\
\text { SphK1 by directly binding to the enzyme and therefore blocks } \\
\text { all of S1P's effects }\end{array}$ \\
\hline Alshaker, $\mathrm{H}$ & 2017 & [34] & NOS-CS & 8 & $\begin{array}{l}\text { FTY720-docetaxel } \\
\text { nanoparticle }\end{array}$ & $\begin{array}{l}\text { FTY720 resensitized breast cancer cells to DTX reducing the } \\
\text { effective dose by four. CNP had the same effect as the control } \\
\text { but lowered the dose needed and reversed the side effects of } \\
\text { weight loss, liver toxicity, and lymphopenia }\end{array}$ \\
\hline Katsuta, E & 2017 & [35] & NOS-CS & 7 & FTY720 & $\begin{array}{l}\text { After treatment with doxorubicin, patient samples showed an } \\
\text { upregulation of STAT3 which was suppressed when } \\
\text { doxorubicin was given with FTY720. Doxorubicin-resistant } \\
\text { breast cancer cell lines showed a high expression of SphK1 } \\
\text { which was inhibited with the help of FTY720 causing a } \\
\text { decrease in growth }\end{array}$ \\
\hline Ochnik, AM & 2017 & [36] & NOS-C & 8 & SKI-II & $\begin{array}{l}\text { Individually both OSI-906 and SKI-II produced positive results } \\
\text { in a dose-dependent manner. Synergism was confirmed by } \\
\text { reducing cell viability better than each individual drug }\end{array}$ \\
\hline \multirow{5}{*}{ Hait, N C } & \multirow{5}{*}{2017} & \multirow{5}{*}{ [37] } & \multirow{5}{*}{ SANRA } & \multirow{5}{*}{9} & SKIII & \\
\hline & & & & & $\mathrm{K}-145$ & $\begin{array}{l}\text { SKI-I, K-145, PF- } 543 \text {, and DMS exerted some beneficial } \\
\text { effects on breast cancer. FTY720 has shown that the }\end{array}$ \\
\hline & & & & & PF-543 & phosphorylated form accumulating in the nucleus inhibits \\
\hline & & & & & DMS & $\begin{array}{l}\text { class IHDACs and can cause ER to form in ER-negative } \\
\text { breast cancer }\end{array}$ \\
\hline & & & & & FTY720 & \\
\hline \multirow{3}{*}{ Tsuchida, J } & \multirow{3}{*}{2017} & \multirow{3}{*}{ [38] } & \multirow{3}{*}{ SANRA } & \multirow{3}{*}{9} & FTY720 & $\begin{array}{l}\text { FTY720 reduced lymphocyte trafficking by binding of the } \\
\text { phosphorylated form to lymphocytes and endothelial cells. }\end{array}$ \\
\hline & & & & & BML-258 & BML-258 decreased growth, size, and metastasis to the lung \\
\hline & & & & & Sonepcizumab & $\begin{array}{l}\text { Sonepcizumab, a monoclonal antibody against } \mathrm{S} 1 \mathrm{P} \text {, reduced } \\
\text { size and proliferation, while a few cases showed complete } \\
\text { disappearance of the tumor }\end{array}$ \\
\hline \multirow[t]{2}{*}{ Nakajima, M } & \multirow[t]{2}{*}{2017} & \multirow[t]{2}{*}{ [5] } & \multirow[t]{2}{*}{ SANRA } & \multirow[t]{2}{*}{9} & FTY720 & $\begin{array}{l}\text { FTY720 reduces expression of S1PR1 while also reducing } \\
\text { overall levels of S1P }\end{array}$ \\
\hline & & & & & SK1-I & SK1-I decreased angiogenesis and lymphangiogenesis \\
\hline Hait, NC & 2015 & [39] & NOS-CS & 7 & FTY720 & $\begin{array}{l}\text { FTY720 is phosphorylated by SphK2 in the nucleus causing an } \\
\text { inhibition of histone deacetylases. This leads to suppression } \\
\text { of tumor growth and development, while also sensitizing } \\
\text { tamoxifen to ER-negative breast cancer }\end{array}$ \\
\hline \multirow{3}{*}{$\begin{array}{l}\text { Mukhopadhyay, } \\
\text { P }\end{array}$} & \multirow{3}{*}{2015} & \multirow{3}{*}{ [6] } & \multirow{3}{*}{ SANRA } & \multirow{3}{*}{7} & SK1-I & SK1-I inhibits angiogenesis and lymphangiogenesis \\
\hline & & & & & PF543 & PF543 is not effective without the presence of TME \\
\hline & & & & & FTY720 & $\begin{array}{l}\text { FTY720 binds to all S1PRs after phosphorylation by SphK2 } \\
\text { except S1PR2. Likewise, it accumulates in the nucleus and } \\
\text { may regulate gene expression of ER }\end{array}$ \\
\hline Marzec, KA & 2015 & [40] & SANRA & 8 & SKI-II & $\begin{array}{l}\text { SKI-II inhibits SphK1, and in combination with gefitinib (EGFR } \\
\text { inhibitor), produced a synergistic effect }\end{array}$ \\
\hline
\end{tabular}




\section{Cureus}

TABLE 2: Compounds/drugs inhibiting the SphK/S1P axis tested in the articles reviewed.

NOS-CS: Newcastle-Ottawa Quality Assessment Scale Cross-Sectional Studies; SANRA: Scale for the Assessment of Narrative Review Articles; NOS-C: Newcastle-Ottawa Quality Assessment Scale Cohort Studies; CSO: coix seed oil; FTY720: fingolimod; TNBC: triple-negative breast cancer; S1PR1: sphingosine-1-phosphate receptor 1; SKI-I: sphingosine kinase inhibitor 1; SKI-II: sphingosine kinase inhibitor 2; SK1-I: sphingosine kinase 1 inhibitor-l; S1PR4: sphingosine-1-phosphate receptor 4; DTX: docetaxel; SphK1: sphingosine kinase 1; S1P: sphingosine-1-phosphate; HDACs: histone deacetylases; ER: estrogen receptor; CNP: complex nanoparticle; S1PRs: sphingosine-1-phosphate receptors; SphK2: sphingosine kinase 2; S1PR2: sphingosine-1-phosphate receptor 2
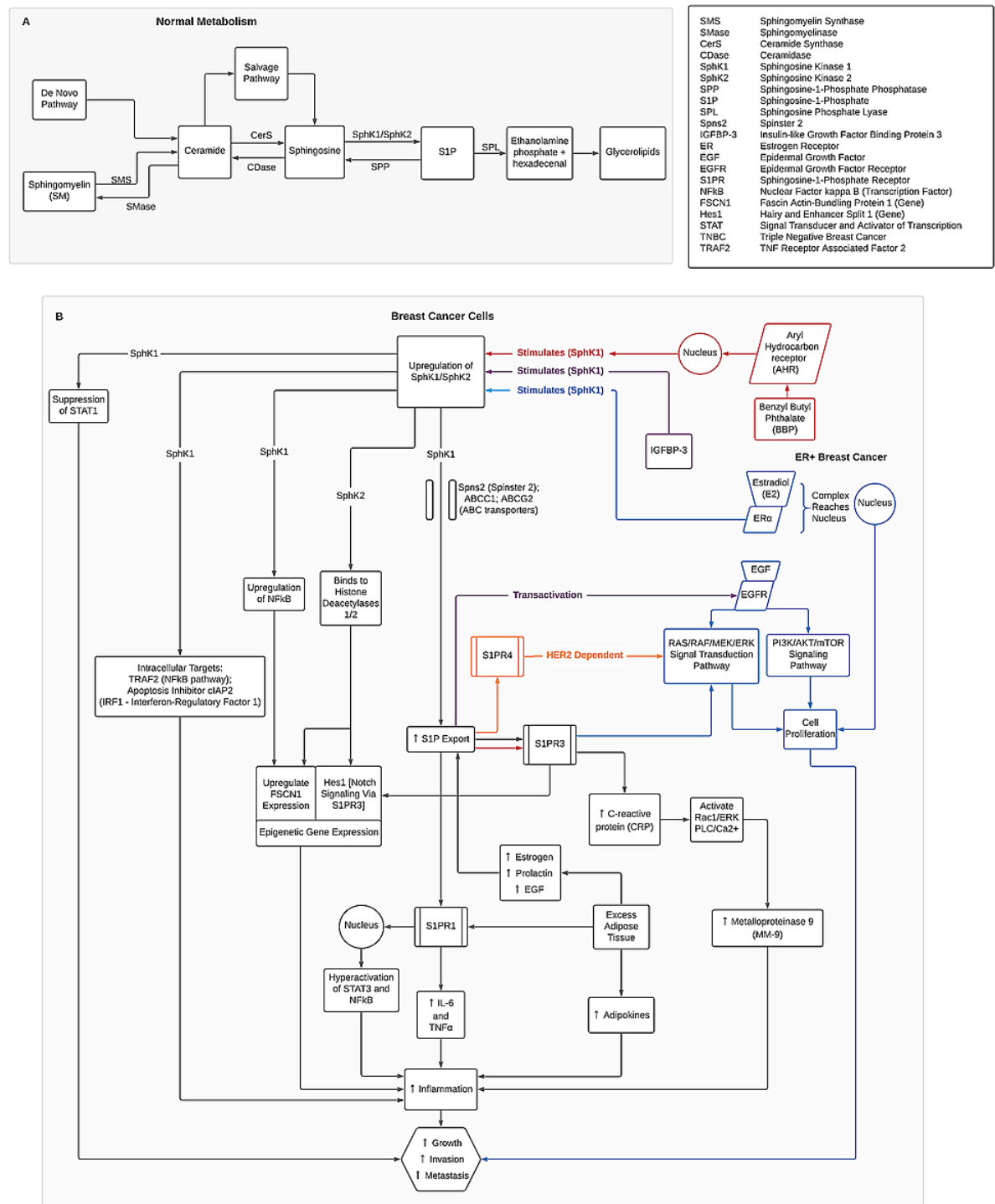

FIGURE 2: (A) Normal metabolism of sphingolipids. (B) Upregulation of SphK/S1P axis in breast cancer cells. 


\section{Cureus}

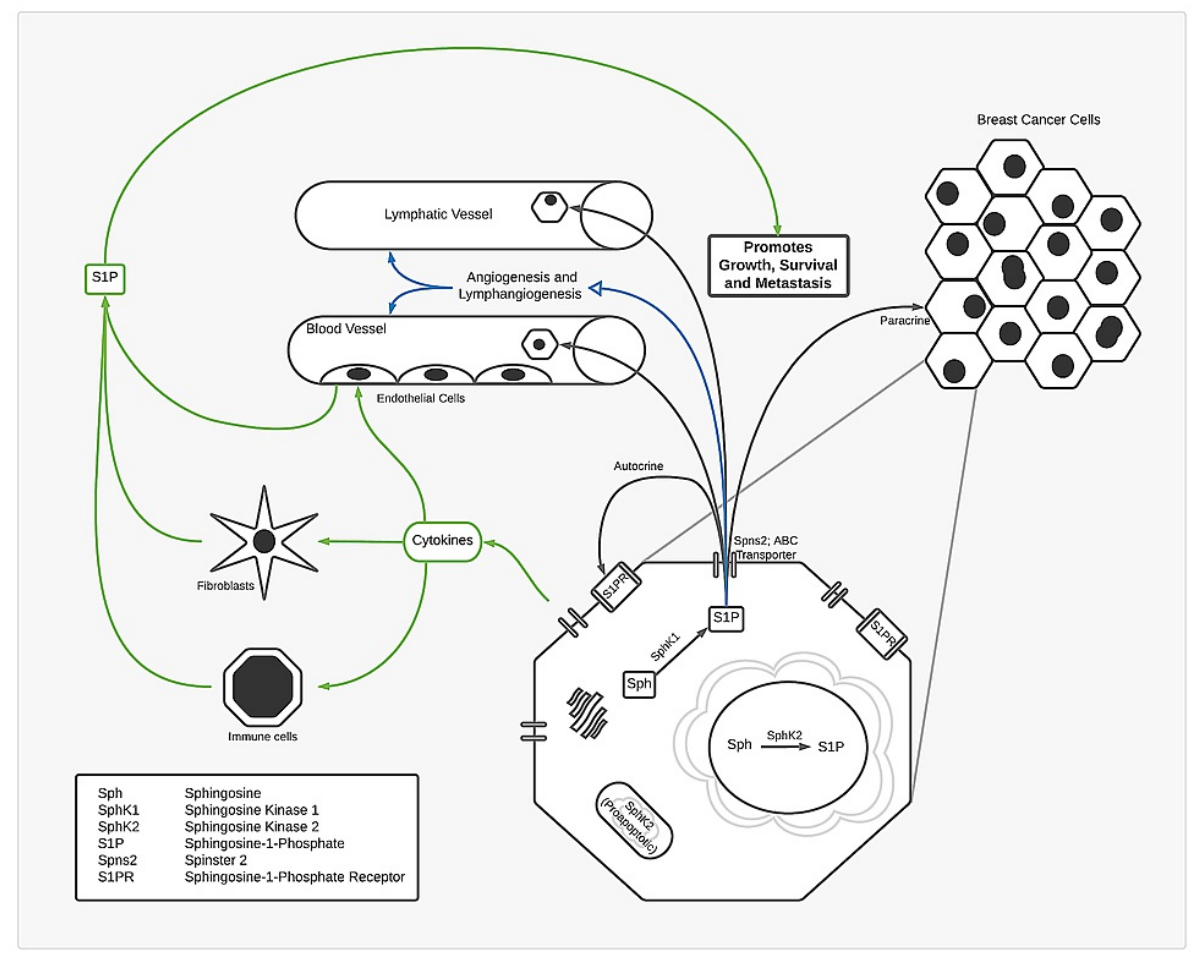

\section{FIGURE 3: Tumor microenvironment: the effect of the upregulation of SphK on the surrounding tumor cells, vessels, and stroma.}

\section{Discussion}

The SphK/S1P axis is a relatively new concept that has the potential to be a factor in the future of breast cancer management. While there are currently modes of treatment available for breast cancer, it is still insufficient in treating complicated and severe cases. The information about the SphK/S1P axis has been summarized below. As new research is published every year, we discuss what is known so far and what information is still needed for future integration into breast cancer management. It is important to note that all studies mentioned were preclinical.

\section{Metabolism of Sphingolipids}

The top part of Figure $2 A$ summarizes the metabolism of sphingolipids in the absence of cancer. Ceramide is a paramount sphingolipid molecule and is formed through many processes [4]. These include de novo synthesis, formation from sphingomyelin, and a particular product in the salvage pathway [37]. The de novo synthesis can occur in the endoplasmic reticulum, lysosomes, and mitochondria, where a series of reactions occur to produce ceramide. Ceramide can also form from sphingomyelin with the help of sphingomyelinase. Likewise, ceramide can form right before the salvage pathway begins. Glucosylceramide, which is formed from ceramide for the salvage pathway by glucosylceramide synthase, can be reversed by glucocerebrosidase. Ceramide is then converted to sphingosine by ceramidase. Sphingosine is also formed indirectly from ceramide via the salvage pathway.

S1P is finally formed from sphingosine by SphK. SphK1 and SphK2 are the two essential isoforms present in different organelles of the cell. SphK2 is present within the nucleus and the mitochondria, while SphK1 is present within the cytosol. S1P is then broken down by sphingosine phosphate lyase (SPL) to form ethanolamine phosphate and hexadecenal, further breaking down into glycerolipids [4]. Some enzymes reverse the process to balance the substrate and product level, keeping the cell environment in homeostasis [37]. These enzymes include sphingomyelin synthase, which converts ceramide back to sphingomyelin, and ceramide synthase (CerS), which converts sphingosine to ceramide. Likewise, sphingosine-1-phosphate phosphatase (SPP) converts S1P back to sphingosine [37].

SphK/S1P Axis in Breast Cancer

SphK, in a typical cell environment, functions in homeostasis. However, in breast cancer cells, studies have shown an upregulation of SphK1/SphK2 [31]. Figure 2B summarizes what is known about the mechanism behind this cascade. The overall outcome is displayed at the bottom of the figure conveying an increase in 
The upregulation of SphK1 has been thoroughly linked with poor prognosis in breast cancer $[8,19,21,24,25]$. SphK1 upregulation causes an increase of S1P in the cytosol. Likewise, the levels of S1P remain high due to the downregulation of SPL. A recent study found breast cancer cells to have low levels of an SPL variant in the cytosol, allowing S1P to stay active [16]. After formation, S1P is excessively exported with the help of specifically identified transporters to the TME. These upregulated transporters include Spinster 2 (Spns2) and specific ATP-binding cassette transporters (ABC transporters), including ABCC1, ABCG2, and ABCC11 [38]. Not all ABC transporters are involved, for example, ABCB1, which does not correlate with increased S1P secretion, breast cancer tumor growth, or angiogenesis [20].

The role of S1P is the cornerstone of breast cancer proliferation. The hyperregulation of S1P has been linked to inflammation, cancer cell survival, tumor growth, metastasis, angiogenesis, lymphangiogenesis, chemokine signaling, epigenetic regulation, and immune cell trafficking [37]. Moreover, a 2020 study determined the increase in S1P due to the upregulation of SphK1 caused an increase in epithelialmesenchymal transition (EMT) [8]. EMT is the loss of differentiation and intercellular junctions, causing the cells to have mesenchymal cell characteristics while being more prone to metastasis.

Once outside the cell, S1P works via autocrine and paracrine action on the S1PRs. As described earlier, there are five subtypes of S1PRs, but not all have been linked to breast cancer promotion. S1PR1, S1PR3, and S1PR4 have been linked to cancer proliferation thus far [3]. Extracellular S1P exerts its inflammatory effects via S1PR1 and S1PR3. The S1P effect on S1PR1 causes an increase in IL-6 and TNF $\alpha$ and increases the activation of signal transducer and activator of transcription 3 (STAT3) and nuclear factor-kappa B (NFkB) within the nucleus, all of which contribute to the inflammation $[6,23]$. Through the effect of S1PR1, obesity can lead to an increase in inflammation and breast cancer proliferation as well [24,32]. The excess adipose tissue increases estrogen, prolactin, and epidermal growth factor (EGF), creating a downstream effect by increasing S1P.

Similarly, there is an increase in adipokines in correlation with obesity, contributing to breast cancer growth. Furthermore, S1P binding to S1PR3 increases inflammation by increasing C-reactive protein (CRP). The increased CRP leads to activation of signaling pathways Rac1/ERK and PLC/Ca2+, thereby causing an upregulation in metalloproteinase 9 (MM-9) expression. Increased MM-9 expression has been linked to increased inflammation as well as increased invasion and metastasis [31]. A study reinforced the importance of S1PR1 and S1PR3 in breast cancer growth and adhesion by silencing the genes for these two receptors, resulting in a statistically reduced growth in the breast cancer tumors [22].

Estrogen receptor (ER) status is another crucial factor regarding the SphK/S1P axis. In ER-positive breast cancer, estradiol (E2) binds to ER $\alpha$, which upregulates SphK1, causing an increase in S1P. Once exported, S1P acts on S1PR3, which stimulates ERK1/2 of the RAS/RAF/MEK/ERK signal transduction pathway leading to cancer cell proliferation [31]. S1P and S1PR3 may also stimulate AKT of the PI3K/AKT/mTOR signaling pathway producing a similar effect [15]. Simultaneously, the E2-ER $\alpha$ complex reaches the nucleus increasing certain transcription factors, and, in turn, promotes breast cancer cell growth. Another study found that high levels of S1PR1 and S1PR3 in ER-positive breast cancer cells resulted in a poor prognosis [22]. This evidence reinforces the importance of ER status in correlation to the SphK/S1P axis.

For ER-negative breast cancer cells, evidence shows a human EGF receptor 2 (HER2)-dependent pathway causes S1PR4 to stimulate the ERK signal pathway. An additional study proved that by downregulating SphK1, the growth of HER2 breast tumors reduced as well. The tumor reduction also correlated with a decrease in claudin-2 expression and may have been an underlying factor [18]. On the other hand, there has also been evidence that S1P levels were lower in the presence of HER2 upregulation [37,38]. The mixture of evidence indicates a need for further research to determine the correlation between HER2 and the SphK/S1P axis.

Concerning EGFR downstream signaling pathways, evidence shows that insulin-like growth factor binding protein-3 (IFGBP-3) upregulates SphK1 [40]. IFGBP-3 essentially uses the same mechanism previously stated (exported S1P acting on the respective receptor) to induce the same signal transduction pathways. The result is an increase in the growth of breast cancer cells and a worse prognosis. This mechanism needs more clarity, though, as one study revealed mixed results. While there was a correlation between gene expression of insulin-like growth factor receptor (IGFR) and SphK1, the protein expression of IGFR and SphK1 did not correlate [36].

S1P not only exerts its effects extracellularly via S1PRs but also intracellularly through different mechanisms. In a recent study, cytosolic S1P generated from SphK1 suppressed signal transducer and activator of transcription 1 (STAT1)-mediated interferon (IFN) signaling to allow excessive growth. STAT1 has shown tumor-suppressing properties, and when SphK1 was decreased, STAT1 signaling increased as well, leading to cell apoptosis [28]. Cytosolic S1P also has intracellular targets that induce inflammation. The targets include a component of the NFkB pathway, TNF receptor-associated factor 2 (TRAF2), as well as an integrant of the interferon regulatory factor 1 (IRF-1) mediator of inflammation, apoptosis inhibitor cIAP2 
[37]. SphK1 regulates other elements as well. Fascin actin-bundling protein 1 (FSCN1) is a gene that promotes metastasis and was shown to upregulate by SphK1 via NFkB in a recent study [13]. Another gene that is upregulated by SphK1 is hairy and enhancer split 1 . It is a component of the Notch signaling pathway whose regulation is mediated through S1PR3 [19,31]. Other genes upregulated by the SphK1/S1P axis in breast cancer have been identified, including CERS1, CERS2, CERS6, and UGCG [11]. Likewise, this epigenetic regulation can also be regulated by SphK2. SphK2 is present in the nucleus where the S1P formed binds to histone deacetylases 1 and 2, essentially inhibiting them. In turn, this epigenetic regulation causes breast cancer progression via histone acetylation and gene transcription [37]. It is important to mention that while upregulation of SphK2 in the nucleus may cause cancer to progress, SphK2 in the mitochondria has the opposite effect. S1P formed in the mitochondria is degraded by SPL to hexadecenal. The product then binds to an apoptosis regulator, promoting the release of cytochrome c [37]. Increased cytochrome c leads to apoptosis and inhibits cancer growth.

Other factors have been discovered to cause an upregulation of SphK1, leading to breast cancer progression. One study identified benzyl butyl phthalate (BBP) promoting breast cancer cell growth by the same mechanism. In a dose-dependent manner, BBP binds to the aryl hydrocarbon receptor (AHR) and stimulates transcription factors, causing an increase in S1P produced by SphK1. S1P subsequently binds to S1PR3 to cause breast cancer growth [27]. A different study decreased lipid concentration in the extracellular matrix to increase its rigidity, resulting in an upregulation of SphK1 [26]. This result increased the metastatic potential and proved to be another factor manipulating the SphK/S1P axis.

SphK1/SphK2 hyperregulation has many downstream consequences that have been identified and replicated. This discussion only took into account what has been published in the last five years. Figure 2 does not cover every detail of the SphK/S1P axis in breast cancer cells, as this concept is intricate. In this section, the intracellular and extracellular ramifications of the SphK/S1P axis were discussed, but the next section considers the larger environment in which the breast tumor thrives.

\section{Tumor Microenvironment}

While understanding the upregulation of the SphK/S1P axis and its sequelae is essential, what is paramount is the environment in which breast cancer cells proliferate. The main components of the TME include blood vessels, lymphatics, and stromal cells. Figure 3 shows a basic summary of the effects of SphK/S1P axis on the TME and how these effects lead to metastasis, angiogenesis, lymphangiogenesis, and immune cell trafficking [37]. Before summarizing the effects of S1P on the TME, appreciating the quotidian environment is vital. S1P levels remain in homeostasis due to the balance between SphK and SPP as well as SPL. While overexpression of SphK can lead to overgrowth and cancer, SPP overexpression can lead to apoptosis [5]. In the absence of cancer, S1P levels in the blood are maintained mainly by erythrocytes and complemented by vascular endothelial cells, according to recent evidence [5]. Endothelial cells of the lymphatics also regulate S1P levels with the help of the Spns2 transporter. Likewise, stromal and immune cells are likely to be a source of production in homeostasis, but more evidence is required.

In the presence of breast cancer cells, S1P levels increase in the TME, causing an imbalance and leading to a cascade of effects. Figure 3 (bottom) represents a breast cancer cell exporting S1P into the TME. The first consequence has been described in detail previously as S1P induces growth and proliferation of the tumor cells and metastasis in an autocrine and paracrine manner via transporters and S1PRs.

The second significant repercussion is on the promotion of angiogenesis and lymphangiogenesis. One study indicated that S1PR1 was responsible for angiogenesis by activating vascular endothelial growth factor receptor, EGFR, and platelet-derived growth factor receptor [3]. It can also be concluded that a vicious cycle can occur because these growth factors can upregulate SphK1. An additional study in 2020 crreated an angiogenesis pathway score utilizing genes associated with S1P to look for a correlation with metastasis, aggressiveness, and inflammation [10]. On the other hand, a recent article studied tumor angiogenesis by contrasting endothelium-dependent vessel (EDV) and vasculogenic mimicry (VM) per S1PR1, the latter associated with a worse prognosis. The study found that by depleting S1PR1, EDV was impaired while VM intensified, leading to a worse prognosis in breast cancer [14]. Another study found similar results in which decreased levels of S1PR1 correlated to a poorer prognosis [9]. Regardless of these results, most research points to a positive correlation between S1P, S1PR1, and angiogenesis. Though there is a large proportion of research regarding angiogenesis, the opposite is true for S1P and lymphangiogenesis [3]. There is plenty evidence regarding metastasis via lymphatics in breast cancer, so lymphangiogenesis can be assumed to have a role, but further research is needed to find the underlying mechanisms.

Lastly, S1P released from breast cancer cells can induce S1P production from non-cancer cells in the TME. This induction is accomplished by increasing cytokines and growth factors mentioned previously, along with fibroblast growth factor (FGF). Along with the cytokines mentioned before, a recent study found a correlation between IL-22 receptor and S1PR1 in highly metastatic, advanced breast cancer tumors [12]. The cells affected by these cytokines and FGF can include endothelial cells, fibroblasts, and immune cells. A study found that breast cancer patients had granulocytes, monocytes, and lymphocytes expressing high levels of S1PRs [23]. An additional study showed that S1PR2 from breast cancer stem cells activated 
fibroblasts [16]. All these cells affected by S1P produce S1P themselves, causing a domino effect increasing survival and growth of the tumor multi-fold.

The combination of effects caused by the SphK1/S1P axis at the cellular level with the surrounding environment is why this mechanism is salient. It has the potential to be a key target for treatment because inhibiting SphK can halt breast cancer progression at each of these levels. The next section discusses the compounds/drugs that inhibit the SphK/S1P axis in one form or another.

Promising Compounds/Drugs

By utilizing the SphK/S1P axis, researchers have tested compounds and drugs that hinder breast cancer tumors. Table 2 summarizes the findings of each study in this review. This aspect is the most novel as no drugs have been used in clinical trials on a large scale. The mentioned studies assess the compounds' efficacy on breast cancer tissue samples, breast cancer cell lines, and mice.

The compounds have been previously classified into SphK inhibitors that inhibit both SphK1 and SphK2 (Pan-SphK inhibitors) and those that selectively inhibit SphK1 [31]. These pan-SphK inhibitors include sphingosine kinase inhibitors 1 and 2 (SKI-I and SKI-II) and N,N-dimethyl-D-erythro-sphingosine (DMS). Most of the other compounds inhibit only SphK1 and include FTY720, PF543, SKI-178, 12aa, SK1-I, SK-A to SK-F, SK1-5C, and BML-258. K-145 inhibits SphK2 and sonepcizumab is the only monoclonal antibody against S1P.

Many studies tested FTY720 (fingolimod) which was approved by the Food and Drug Admininstration for use in multiple sclerosis previously [3]. FTY720 has been shown to bind to SphK1, blocking S1P formation in the cytosol directly [3]. On reaching the nucleus of a breast cancer cell, it is phosphorylated by SphK2, inhibiting histone deacetylases. This inhibition causes an upregulation of genes concerned with ER, which otherwise have been suppressed in ER-negative breast cancer [3,6,30,37,39]. FTY720 was also shown to inhibit obesityrelated inflammation, proliferation, and metastasis by inhibiting S1PR1 [29,40]. One study found that the phosphorylated FTY720 also reduced lymphocyte trafficking by binding to lymphocytes and endothelial cells, essentially affecting the TME [38]. This drug has been shown to inhibit the growth of breast cancer cells but with limited efficacy alone [35]. Studies have shown that in combination with doxorubicin or docetaxel (DTX), the dose needed for each drug was reduced, while the incidence of side effects of weight loss, liver toxicity, and lymphopenia also reduced [28,31,34]. The mechanism of FTY720 is well established and has been proven to reduce breast tumor size and growth in coordination with other modes of treatment for breast cancer. Moving on to more extensive studies with more subjects or even randomized control trials may be the next step.

Other compounds inhibiting SphK1 include PF543, which has been shown to reduce invasiveness and metastasis of breast cancer tumors; however, one study found that PF543 is not efficacious without the presence of the TME [6]. The result indicates the need to test these compounds in vivo amply to see the overall effect. In a separate study, PF453 was proven to improve doxorubicin sensitivity in breast cancer cells, further demonstrating its efficacy [28]. SK1-I is another selective SphK1 inhibitor that has been shown to reduce angiogenesis and lymphangiogenesis [5,6,31]. SK1-I was another compound that inhibited the growth of breast cancer tissue via the TME. SK-F is a recently discovered compound with a similar mechanism, which showed no statistically significant change in tumor proliferation individually, but decreased tumor size in the presence of DTX at lower doses [33]. Further studies in the future may give more insight into this compound. BML-258 has been shown to inhibit SphK1 and decrease growth, size, and metastasis of the breast tumors [38]. SK1-5C has shown similar results by decreasing ERK1/2 and AKT pathways.

Similarly, the pan-SphK inhibitors, SKI-I and SKI-II, and DMS have been beneficial in breast cancer inhibition. SKI-I was shown to reduce the tumor growth without a high degree of side effects [31]. SKI-II was tested in combination with EGFR inhibitor, gefitinib, and a dual IGF1R/insulin receptor tyrosine kinase inhibitor, OSI-906, in two separate studies. Each study resulted in a synergistic effect in a dose-dependent manner $[36,40]$. K-145 was the only selective SphK2 inhibitor mentioned in the reviewed studies showing beneficial effects [37]. Likewise, the only monoclonal antibody against S1P was sonepcizumab, which also reduced growth and size, while, in some cases, caused a complete disappearance of the breast tumor [38]. One study analyzed coix seed oil (CSO) and showed that it decreased the expression of S1PR1, cyclin D1, phosphorylated MAPK, and AKT while at the same time upregulating p27. The effect of CSO caused a decrease in viability and inhibited the growth of the breast cancer cell and induced apoptosis [29]. Although these compounds have shown value in different aspects, more studies are required to prove whether they can be used to manage breast cancer in the future.

With the research and information discussed, these compounds have potential but they lack clinical evidence. While many studies have investigated some compounds, many still lack collective evidence. Still, these compounds are promising, especially when complimenting other drugs. Where modern treatment stops, this mechanism can eventually have an impact if it reaches clinical trials. Currently, there are no active clinical trials pertaining to any SphK inhibitors and their impact in breast cancer [41]. 
While we strive for the most up-to-date and accurate information, there are still limitations concerning this review. First, while the respective checklist assessed each study for quality, our process was still subjective. There is a possibility that some articles were overlooked, which could have accentuated this review.

Likewise, articles from only the last five years were screened to collate the information presented. There may have been information from older articles that may have underscored an idea or filled the information gap. Moreover, most of the studies only had samples of cancerous breast tissue available for analysis. The studies were not done on actual patients, and therefore, side effects and overall quality of life were not assessed. The actual efficacy and adverse effects of the compounds mentioned are yet to be determined.

\section{Conclusions}

The purpose of the study was to summarize the literature concerned with the SphK/S1P axis in breast cancer. We looked at the physiology of sphingolipid metabolism before describing what occurs in breast cancer cells. The focus then shifted to the effects the tumor had on the TME due to its importance in tumor survival. Lastly, we analyzed the compounds acting on SphK1 and SphK2.

While summarizing the SphK/S1P axis, we found that more research is needed. In our opinion, areas that need to be emphasized include identifying and solidifying the exact role of the SphK/S1P axis at the every level from genetic and intracellular expression to the TME. Specifically, more studies are needed to understand lymphangiogenesis in regards to SphK. Likewise, testing the discovered compounds and drugs at the next stage is also essential. It was shown that SphK inhibitors could have a synergistic action with other drugs on the market. Clinical trials are needed to dissect these compounds thoroughly. On the same note, nanoparticles carrying a combination of therapy for breast cancer is novel and needs further evaluation. By compiling the information thus far, we hope that our effort is a stepping stone for eventual clinical research.

\section{Additional Information}

\section{Disclosures}

Conflicts of interest: In compliance with the ICMJE uniform disclosure form, all authors declare the following: Payment/services info: All authors have declared that no financial support was received from any organization for the submitted work. Financial relationships: All authors have declared that they have no financial relationships at present or within the previous three years with any organizations that might have an interest in the submitted work. Other relationships: All authors have declared that there are no other relationships or activities that could appear to have influenced the submitted work.

\section{References}

1. Siegel RL, Miller KD, Jemal A: Cancer statistics. CA Cancer J Clin. 2017, 67:7-30. 10.3322/caac.21387

2. Breast cancer facts. (2020). Accessed: November 20, 2020: https://www.nationalbreastcancer.org/breastcancer-facts.

3. Geffken K, Spiegel S: Sphingosine kinase 1 in breast cancer. Adv Biol Regul. 2018, 67:59-65. 10.1016/j.jbior.2017.10.005

4. Singh SK, Spiegel S: Sphingosine-1-phosphate signaling: a novel target for simultaneous adjuvant treatment of triple negative breast cancer and chemotherapy-induced neuropathic pain. Adv Biol Regul. 2020, 75:100670. 10.1016/j.jbior.2019.100670

5. Nakajima M, Nagahashi M, Rashid OM, Takabe K, Wakai T: The role of sphingosine-1-phosphate in the tumor microenvironment and its clinical implications. Tumour Biol. 2017, 39:1010428317699133. $10.1177 / 1010428317699133$

6. Mukhopadhyay P, Ramanathan R, Takabe K: S1P promotes breast cancer progression by angiogenesis and lymphangiogenesis. Breast Cancer Manag. 2015, 4:241-244. 10.2217/bmt.15.20

7. Sukocheva OA, Furuya H, Ng ML, et al.: Sphingosine kinase and sphingosine-1-phosphate receptor signaling pathway in inflammatory gastrointestinal disease and cancers: a novel therapeutic target. Pharmacol Ther. 2020, 207:107464. 10.1016/j.pharmthera.2019.107464

8. Chen Z, Liu B: Sphk1 participates in malignant progression of breast cancer by regulating epithelialmesenchymal transition and stem cell characteristics. Tissue Cell. 2020, 65:101380. 10.1016/j.tice.2020.101380

9. Kim EY, Choi B, Kim JE, Park SO, Kim SM, Chang EJ: Interleukin-22 mediates the chemotactic migration of breast cancer cells and macrophage infiltration of the bone microenvironment by potentiating S1P/SIPR signaling. Cells. 2020, 9:131. 10.3390/cells9010131

10. Oshi M, Newman S, Tokumaru Y, et al.: Intra-tumoral angiogenesis is associated with inflammation, immune reaction and metastatic recurrence in breast cancer. Int J Mol Sci. 2020, 21:6708. 10.3390/ijms21186708

11. Sakharkar MK, Kaur Dhillon S, Chidambaram SB, Essa MM, Yang J: Gene pair correlation coefficients in sphingolipid metabolic pathway as a potential prognostic biomarker for breast cancer. Cancers (Basel). 2020, 12:1747. 10.3390/cancers12071747

12. Zhong L, Xie L, Yang Z, Li L, Song S, Cao D, Liu Y: Prognostic value of S1PR1 and its correlation with immune infiltrates in breast and lung cancers. BMC Cancer. 2020, 20:766. 10.1186/s12885-020-07278-2

13. Acharya S, Yao J, Li P, et al.: Sphingosine kinase 1 signaling promotes metastasis of triple-negative breast cancer. Cancer Res. 2019, 79:4211-4226. 10.1158/0008-5472.CAN-18-3803

14. Liu S, Ni C, Zhang D, et al.: S1PR1 regulates the switch of two angiogenic modes by VE-cadherin phosphorylation in breast cancer. Cell Death Dis. 2019, 10:200. 10.1038/s41419-019-1411-X 
15. Alshaker H, Wang Q, Brewer D, Pchejetski D: Transcriptome-wide effects of sphingosine kinases knockdown in metastatic prostate and breast cancer cells: implications for therapeutic targeting. Front Pharmacol. 2019, 10:303. 10.3389/fphar.2019.00303

16. El Buri A, Adams DR, Smith D, Tate RJ, Mullin M, Pyne S, Pyne NJ: The sphingosine 1-phosphate receptor 2 is shed in exosomes from breast cancer cells and is N-terminally processed to a short constitutively active form that promotes extracellular signal regulated kinase activation and DNA synthesis in fibroblasts. Oncotarget. 2018, 9:29453-29467. 10.18632/oncotarget.25658

17. Engel N, Adamus A, Frank M, et al.: First evidence of SGPL1 expression in the cell membrane silencing the extracellular S1P siren in mammary epithelial cells. PLoS One. 2018, 13:196854. 10.1371/journal.pone.0196854

18. Shimizu Y, Furuya H, Tamashiro PM, et al.: Genetic deletion of sphingosine kinase 1 suppresses mouse breast tumor development in an HER2 transgenic model. Carcinogenesis. 2018, 39:47-55. 10.1093/carcin/bgx097

19. Wang S, Liang Y, Chang W, Hu B, Zhang Y: Triple negative breast cancer depends on sphingosine kinase 1 (SphK1)/sphingosine-1-phosphate (S1P)/sphingosine 1-phosphate receptor 3 (S1PR3)/notch signaling for metastasis. Med Sci Monit. 2018, 24:1912-1923. 10.12659/msm.905833

20. Yamada A, Nagahashi M, Aoyagi T, et al.: ABCC1-exported sphingosine-1-phosphate, produced by sphingosine kinase 1, shortens survival of mice and patients with breast cancer. Mol Cancer Res. 2018, 16:1059-1070. 10.1158/1541-7786.MCR-17-0353

21. Do SI, Kim HS, Kim K, et al.: Predictive and prognostic value of sphingosine kinase 1 expression in patients with invasive ductal carcinoma of the breast. Am J Transl Res. 2017, 9:5684-5695.

22. Calis IU, Cosan DT, Mutlu F: Effects of S1P1 and S1P3 in ER(+) and ER(-) breast cancer cells . Anticancer Res. 2017, 37:5469-5475. 10.21873/anticanres.11976

23. Maia LP, Santos PS, Alves PT, et al.: Altered leukocyte sphingolipid pathway in breast cancer. Int J Mol Sci. 2017, 18:2521. 10.3390/ijms18122521

24. Nazouri AS, Asadpour O, Dabiri S, Pourseyedi B, Lashkarizadeh MR, Zianalinejad H: High expression of sphingosine kinase 1 in estrogen and progesterone receptors-negative breast cancer. Iran J Pathol. 2017, 12:218-224. 10.30699/IJP.2017.25049

25. Zhu YJ, You H, Tan JX, et al.: Overexpression of sphingosine kinase 1 is predictive of poor prognosis in human breast cancer. Oncol Lett. 2017, 14:63-72. 10.3892/ol.2017.6134

26. Ko P, Kim D, You E, et al.: Extracellular matrix rigidity-dependent sphingosine-1-phosphate secretion regulates metastatic cancer cell invasion and adhesion. Sci Rep. 2016, 6:21564. 10.1038/srep21564

27. Wang YC, Tsai CF, Chuang HL, Chang YC, Chen HS, Lee JN, Tsai EM: Benzyl butyl phthalate promotes breast cancer stem cell expansion via SPHK1/S1P/S1PR3 signaling. Oncotarget. 2016, 7:29563-29576. 10.18632/oncotarget.9007

28. Hii LW, Chung FF, Mai CW, et al.: Sphingosine kinase 1 regulates the survival of breast cancer stem cells and non-stem breast cancer cells by suppression of STAT1. Cells. 2020, 9:886. 10.3390/cells9040886

29. Fang T, Jiang YX, Chen L, et al.: Coix seed oil exerts an anti-triple-negative breast cancer effect by disrupting miR-205/S1PR1 axis. Front Pharmacol. 2020, 11:529962. 10.3389/fphar.2020.529962

30. Rupp T, Pelouin O, Genest L, Legrand C, Froget G, Castagné V: Therapeutic potential of Fingolimod in triple negative breast cancer preclinical models. Transl Oncol. 2021, 14:100926. 10.1016/j.tranon.2020.100926

31. Alshaker H, Thrower H, Pchejetski D: Sphingosine kinase 1 in breast cancer-a new molecular marker and a therapy target. Front Oncol. 2020, 10:289. 10.3389/fonc.2020.00289

32. Nagahashi M, Yamada A, Katsuta E, et al.: Targeting the SphK1/S1P/S1PR1 axis that links obesity, chronic inflammation, and breast cancer metastasis. Cancer Res. 2018, 78:1713-1725. 10.1158/0008-5472.CAN-171423

33. Alshaker H, Srivats S, Monteil D, Wang Q, Low CMR, Pchejetski D: Field template-based design and biological evaluation of new sphingosine kinase 1 inhibitors. Breast Cancer Res Treat. 2018, 172:33-43. 10.1007/s10549-018-4900-1

34. Alshaker H, Wang Q, Srivats S, Chao Y, Cooper C, Pchejetski D: New FTY720-docetaxel nanoparticle therapy overcomes FTY720-induced lymphopenia and inhibits metastatic breast tumour growth. Breast Cancer Res Treat. 2017, 165:531-543. 10.1007/s10549-017-4380-8

35. Katsuta E, Yan L, Nagahashi M, et al.: Doxorubicin effect is enhanced by sphingosine-1-phosphate signaling antagonist in breast cancer. J Surg Res. 2017, 219:202-213. 10.1016/j.jss.2017.05.101

36. Ochnik AM, Baxter RC: Insulin-like growth factor receptor and sphingosine kinase are prognostic and therapeutic targets in breast cancer. BMC Cancer. 2017, 17:820. 10.1186/s12885-017-3809-0

37. Hait NC, Maiti A: The role of sphingosine-1-phosphate and ceramide-1-phosphate in inflammation and cancer. Mediators Inflamm. 2017, 2017:4806541. 10.1155/2017/4806541

38. Tsuchida J, Nagahashi M, Takabe K, Wakai T: Clinical impact of sphingosine-1-phosphate in breast cancer . Mediators Inflamm. 2017, 2017:2076239. 10.1155/2017/2076239

39. Hait NC, Avni D, Yamada A, et al.: The phosphorylated prodrug FTY720 is a histone deacetylase inhibitor that reactivates ER $\alpha$ expression and enhances hormonal therapy for breast cancer. Oncogenesis. 2015, 4:156. 10.1038/oncsis.2015.16

40. Marzec KA, Baxter RC, Martin JL: Targeting insulin-like growth factor binding protein-3 signaling in triplenegative breast cancer. Biomed Res Int. 2015, 2015:638526. 10.1155/2015/638526

41. Search results in the National Library of Medicine for sphingosine kinase . (2020). Accessed: February 15, 2021: https://www.clinicaltrials.gov/ct2/results?term=sphingosine+kinase. 